\title{
Performance Analysis \& Comparison of Optimal Economic Load Dispatch using Soft Computing Techniques
}

\author{
Vijay Kumar, Associate Professor, \\ Adesh Institute of Technology, \\ Kharar (Mohali), India
}

\author{
Dr. Jagdev Singh, Associate Professor, \\ Beant College of Engineering \& Technology, \\ Gurdaspur, India
}

\author{
Dr. Yaduvir Singh, \\ Harcourt Butler Technological Institute, \\ Kanpur, India
}

\author{
Dr. Sanjay Prakash Sood, Associate Professor, \\ Centre for development \& advanced computing, \\ Mohali, India
}

\begin{abstract}
Power plants not situated at similar space from center of loads and their fuel prices are dissimilar. In this paper, ELD of actual power generation measured.ELD is preparation of generators to reduce total functioning price of generator units exposed to equality constraint of power balance within minimum and maximum working limits of producing units. In this paper FL, GAs \& hybridization of GA-FL is utilized to find optimal solution of ELD systems. ELD resolutions found by resolving conservative load flow equations though at same time reducing fuel prices. Performance of results is analyzed by comparing the data values obtained with the help of soft computing techniques in ELD.
\end{abstract}

Keywords-ELD; FL; GA; FCGA

\section{INTRODUCTION}

ED is small term fortitude of optimal output of a lot of electricity generation amenities \& to meet system load at nethermost probable cost subject to transmission and working restraints.ELD is resolved by particular computer software which honor operational and system restraints of existing possessions and consistent transmission abilities. In electrical power systems, a incessant equilibrium must be preserved among electrical generation and fluctuating load mandate, though system frequency, voltage stages and security too reserved constant. Besides, it is required that price of such generation be less. In addition, separation of load in producing plant develops a vital operation in addition to an economic matter which resolved at each load change $(1 \%)$ or each $2-3$ minutes. Research methods effectively castoff to resolve optimum load flow difficulties by using direct or non-linear encoding but these algorithms usually restricted to systematic functions. Numerous tasks are multi-modal, intermittent. Stochastic selection approaches used to improve these functions. Although customary resolution methods use features of problem to govern following sampling point, stochastic resolution methods make no such conventions. In its place, subsequent sampled point resolute centered on stochastic sampling or decision rules than a set of deterministic conclusion rules. GAs used to resolve tough difficulties with objective functions that not retain possessions such as continuity, differentiability. These algorithms preserve and operate a set of answers and implement a existence of the fittest approach in their hunt for a improved solution.

The process of generation services to harvest energy at deepest price to constantly assist clients by identifying any working limits of generation and transmission services. Planning and process of power systems under prevailing circumstances, its growth and future growth requires load flow educations, short circuit studies and constancy studies. Though load flow studies are very important for planning, control and processes of prevailing \& future growth as acceptable operation of the system be contingent upon knowing belongings of inter connection, new loads, new producing stations or new transmission lines afore they are installed.

\section{LITERATURE SURVEY}

G.Sahu et.al (2014) grants the presentation of GA to resolve ELD problem of power system. Planned algorithm verified on two dissimilar test systems seeing transmission damages. The chief cause of cutting total fuel cost and preserve power flows within safety range. B. Hosamani et.al (2014) addresses planning and operation of power systems and introduced an approach to provide consumers with reliable and quality power at an economical cost. The main intent of this paper is to develop efficient and fast Fuzzified Particle Swarm Optimization (FPSO) procedure to attain the optimum results of multi-constrained dynamic ED, OPF. This paper focuses on the multi- constrained dynamic economic dispatch problem and introduces the Fuzzified particle swam optimization to solve it. S. H.Elyas et.al (2014) introduces a proficient approach for comprehending problem of ELD with effect of valve point by utilizing a novel hybrid procedure for optimization. The fundamental goal for discovery resolution of problem of ELD is to plan the output of committed units of generator so as to fulfill the load of system under numerous operating imperatives. B.Sahu (2013) explains GA and quadratic programming ideas in resolving economic load dispatch (ELD) in which entire price of producing power is reduced with a valve point loading outcome though satisfying load request regardless of transmission line losses. V. Karthikeyan (2013) 
explains that price competence is utmost significant problem of power system operations and makes an attempt to catch out the least price by using Particle Swarm Optimization Procedure considering statistics of three generating units. The paper involves the use of damage coefficients with maximum power limit and cost function. PSO and Simulated Annealing smeared to find out the least price for diverse power request. $\mathbf{F}$. Farheen et.al (2013) presented operation of PSO for dynamic problem of ELD. The economic operation of the systems which are generating has constantly involved an imperative position in an industry of electric power. A.Gharegozi et.al (2013) presents a novel method to solve the issue of best planning using Cuckoo Search Algorithm. The proposed approach provides the most appropriate convergence in the response, high computational speed and high accuracy. L. Chopra et.al (2012) shows simple GA and refined GA technique applicable to ELD which books for reduction of price for operational constraints. Lambda iteration technique needs precise alteration of lambda which not provide global optimum resolution. A.Hasan Zade et.al (2011) describes ELD problem as a constrained optimization problem and hence efficient method needed to explain this problematic. The paper involves use of a particular variant of Evolutionary Algorithm namely DE to address ELD problem. Fuzzy logic controller designed to control the amplification factor vector of $\mathrm{DE}$ dynamically during the process of optimization. S.C. Swain et.al (2010) introduces the utilizations of techniques of computational intelligence to problems of ELD. The equation of fuel price of thermal plant is normally communicated a quadratic equation which is continuous. In conditions of real world, the equations of fuel cost occur discontinuous. In perspective of above, both cost equations which are continuous or discontinuous are considered in the present paper.Bakirtzis. A (1994) presented two GAs results to ELD. Benefit of GA resulting not enforce any convexity limitations on generator price function. Additional benefit for GAs for efficiently coded to effort on parallel machines. A.G. Bakirtzis et.al (2002) introduces an enhanced genetic algorithm for finding the solution of optimal power flow (OPF) with control variables which are both discrete and continuous. H.Jagabondhu et.al (2009) grants a relative learning of four diverse evolutionary algorithms i.e. GA bacteria foraging optimization, ant colony optimization and PSO for solving ELD. Concert of every algorithm for resolving ED problem analysed and simulation consequences are shown for precision, consistency and finishing time. Altun $\mathbf{H}$ et.al (2008) highlighted execution issues of soft computing methods for a effective solicitation to solve ED problem for inhibited optimization problem in power systems. The paper presents review of fundamentals of the methods and includes discussion of the implementation of methods in ED problematic. A.Kandari et.al (2007) grants a novel and precise technique for assessing input-output curve limits of power plants. These limits are very imperative for execution economic dispatch values. The greater the precision of projected coefficients, extra precise consequences attained from calculations of economic dispatch. J. Nanda (2001) resolves ELD problematic with Line flow limits over operative application of GA since losses of system transmission, power balance equation as equality constraint, active power generations of units and current limits in different lines for inequality constraints.Palanichamy et.al (1991) offered a direct and computationally effective technique for ELD. The accustomed B coefficients used for calculation of transmission losses, incremental transmission losses and penalty factors.

\section{ECONOMIC LOAD DISPATCH}

ELD is one of substantial functions in automatic generation control. The Economic Load Dispatch of power production units is an important issue in electric utility industry. Schedule of individual units production which reduces total functioning price of a power system while meeting total load plus transmission losses in generator parameters. This is implication to save energy and tumbling emission.

The problematic goes more compound in great scale system henceforth problematic to find out optimum result due to nonlinear function which comprises number of local optimum. Therefore, importance to resolve this problematic precisely. For financial operation of system total ultimatum must be optimally collective between all generating units reducing total production rate though sustaining operative limits on system.

The fuel rate for all power generation unit explained firstly. Hence total production cost function of ELD problem is distinct as the whole sum of the fuel prices for all generating plant units as stated below:

$$
\mathrm{F}_{\mathrm{T}}=\sum_{i=1}^{N G}\left\{a_{i} P_{i}^{2}+b_{i} P_{i}+c_{i}\left|d_{i} \operatorname{sine}_{i}\left(P_{i}^{\min }-P_{i}\right)\right|\right\},
$$

Where, NG is total number of producing units

$\mathrm{F}_{\mathrm{T}}$ is total generating price

$\mathrm{P}_{\mathrm{i}}$ is the power output of producing unit $\mathrm{i}$

$\mathrm{P}_{\mathrm{i}}^{\mathrm{min}}$ is lowest output of producing unit $\mathrm{i}$

$a_{i}, b_{i}, c_{i}, d_{i}, e_{i}$ are fuel rate coefficients of unit $i$

Above equation calculates total generating rate of producing plant.

\section{A. The Lambda-Iteration Method (LIM)}

This is utmost prevalent technique to contract with ELD problematic. Here, variable introduced for solving constraint optimization problem which is said to be as Lagrange multiplier. Lambda explained by unraveling systems of equation. All inequality constraints gratified equations resolved by iterative technique

1) Let a appropriate value $\lambda(0) \&$ this value must be greater than prevalent capture of incremental rate distinctive of the numerous generators.

2) Calculate the separate generations

3) Check the equality

$\left.\operatorname{Pd}=\sum_{n=1}^{n} P n\right)$

\section{B. Langrangian multiplier}

Significant and simplest technique of stating economic dispatch load method as a transmission loss of generator power output is B- coefficients. The general formulae of loss formulae as:

$P_{L}=\sum_{i=1}^{k} \sum_{j=1}^{k} P_{i} B_{i j} P_{j}$ 
Bij are loss coefficients or B-coefficients, Pi ,PJ are real power injection of ith, jth buses.

Above inhibited optimization problematic changed into unrestricted optimization problem. Langrangian multiplier is a method applied to minimize or maximize in equality constraints form. An augmentes function is as:

$\mathrm{L}=C_{t}+\lambda\left(P_{D}+P_{L}-\sum_{i=1}^{k} P_{i}\right)$

$\mathrm{Ct}$ is total fuel cost for all generating units and $\lambda$ is said as Langranian multiplier. For minimizing the cost, $\mathrm{L}$ with $\mathrm{Pi}$ equal to zero.

\section{Fuzzy Logic in ELD}

The characteristic of fuzzy logic to deal with fuzzy or crisp values without much concern for precise input and continuous operation in case of feedback sensor failure makes it be called as robust and reliable method. Despite of wide variation in the inputs output control is smooth control function. The fuzzy logic controller operates on user-defined rules hence it adapted modify system performance. By the generation of appropriate governing rules the system can be incorporated with new sensors. Fuzzy logic is not limited one or two control outputs, and it is not essential to calculate rate-of-change of limits in order for implementation. The sensor data is sufficient because that provides some signal of system action.

The fuzzy modeling control system starts with deciding input and output variables of fuzzy logic. Mamdani fuzzy inference system used for this. This method used for fuzzy modeling process to acquire information about a data set to calculate the membership function limits which greatest permit the related fuzzy inference system to trail the specified inputoutput data. This FIS system intended for MISO system. MISO system comprises two inputs and one output.

\section{Linguistic variables}

These are variables stated in plain language words. These show an significant role in demonstrating crisp information such that it accurately suitable for the problem. Since usage of linguistic variables detected to decrease whole computation complication in many applications \& mainly valuable in representing composite non-linear applications.Linguistic variables are fundamental to fuzzy logic operations, however they are frequently flouted in the arguments on the virtues of fuzzy logic. In fuzzy logic applications, non-numeric linguistic variables frequently applied relative to arithmetical values.

\section{RESEARCH METHODOLOGY}

\section{A. Algorithm for ELD Using GA}

The numerous stages included in solution of GA Algorithm are:

1) Select Population extent, amount of generations, substrings length and quantity of trials.

2) Produce primary arbitrarily coded strings as population associates in the first generation.

3) Decipher population to acquire power generation of units in strings.
4) Implement load flow reflecting the unit generation excluding for the slack bus. To assess the system transmission losses, slack bus generation, line flows.

5) Calculate fitness of population members.

6) Execute selection centered on Reproduction executing. Roulette Wheel process with embedded Elitism trailed by crossover with embedded .Mutation to produce the novel population for the subsequent generation.

\section{B. Algorithm for ELD Using GA}

Lambda iteration method decent loom to determine ELD due to which generator parameters easily controlled. The penalty factors applied to reflect the impact of losses. Fuzzy with ELD system is applicable to optimization problems. System will obtain optimum resolution to complications with fuzzy constrictions and fuzzy variables. The values of power which is obtained from ELD is then adjusted by fuzzy practical rules and gives more appropriate and approximate results for power of each unit.

- Compute optimal dispatch and entire rate ignoring losses $\lambda$.

- Use dispatch and loss formula, compute system losses. It explained by using MATLAB script and function file that use fsolve MATLAB function to resolve system of equations.

- Discover optimal dispatch for a entire generation of PD by coordination equations and loss formula.

- Economic dispatch problematic containing transmission losses computed for effect of transmission losses to represent total transmission loss as a quadratic function of generator power outputs.

- For minimum cost derivative of $\mathrm{L}$ (penalty factor) is required with each Pi equal to zero.

- For generating unit outputs P1, P2 and P3are power units of 1, 2 and 3 for better approximation. It can be designed as $\mathrm{s}$ flowchart as:

if $\mathrm{P} 3<\mathrm{P} 3 \mathrm{~min} \mathrm{P} 3=\mathrm{P} 3 \mathrm{~min}$; else

if $\mathrm{P} 3>\mathrm{P} 3 \max \mathrm{P} 3=\mathrm{P} 3 \max$; end end if $\mathrm{P} 2<\mathrm{P} 2 \min \mathrm{P} 2=$ $\mathrm{P} 2 \mathrm{~min}$; else if $\mathrm{P} 2>\mathrm{P} 2 \mathrm{max} \mathrm{P} 2=\mathrm{P} 2 \mathrm{max}$; end end if $\mathrm{P} 1<\mathrm{P} 1 \mathrm{~min}$ $\mathrm{P} 1=\mathrm{P} 1 \mathrm{~min}$; else if $\mathrm{P} 1>\mathrm{P} 1 \mathrm{max} \mathrm{P} 1=\mathrm{P} 1 \mathrm{max}$;

- Units are ranked according to their full load production cost rate and committed accordingly.

- The system obtain optimal solution to problems with fuzzy constraints and fuzzy variables.

\section{Algorithm for ELD Using Fuzzy-GA}

1) First of all, adjust all limits population size, number of generations,sub-strings length.

2) Initial arbitrary population of individuals produced where the entities coded string of binary numerals.

3) Assessment of fitness population members accomplished on the basis of a fitness function. 
4) When optimization benchmark happened, nominated population members more accepted on for diverse processes.

5) The assortment procedure is achieved.

6) Afterward, fuzzy logic originates into play with fuzzy crossover regulator.

7) The random member first associated with the crossover probability value and if benchmark gratified, border achieved.

8) Likewise for mutation, arbitrary member equated beside mutation probability value.

\section{EXPERIMENTAL ANALYSIS \& RESULTS}

\section{A. Results obtained for ELD using Genetic Algorithm}

1) Power Generated

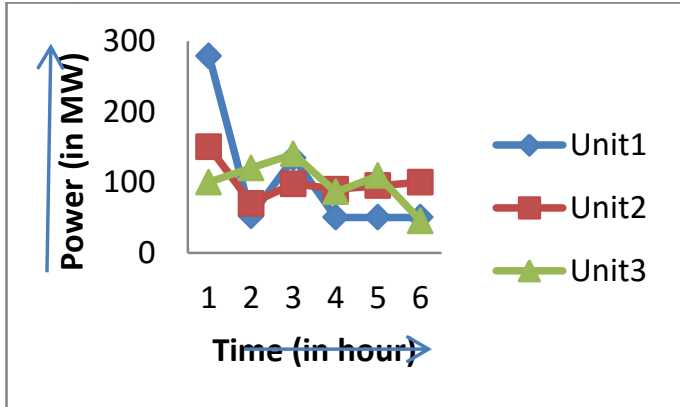

Fig. 1. Power generated

Figure 1 shows power generation for three units which varying according to time/hour.In unit-1 range of powers are 278.912,52.260,134.305,50,50 \& 50 for different ranges which shown by blue lines in figure. In unit-2, power ranges are 150 , $70,97.77,90,95 \& 100$ for power ranges shown by red lines .In unit -3, power ranges are 100, 120, 140, 86.23, 109.30 \& 45 for different maximum \& minimum power ranges \& shown by green lines in figure.All power are in MW.

\section{2) Fuel Cost}

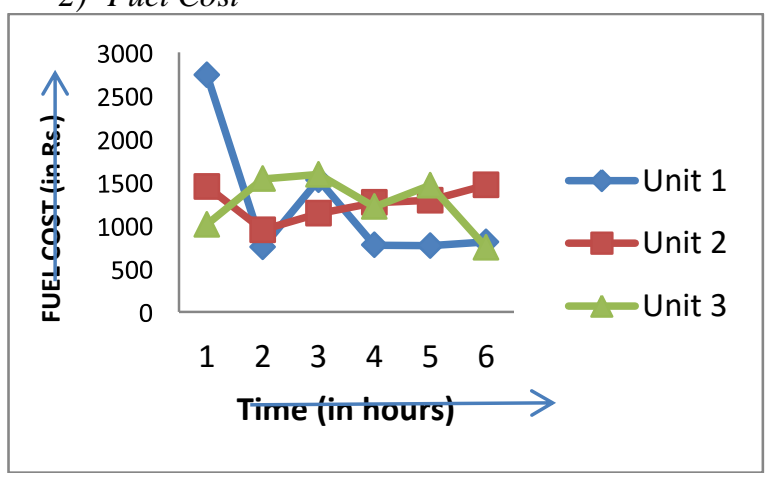

Fig. 2. Fuel Cost

Figure 2 describes fuel cost for three units \& cost is according to time per hour.For unit 1 fuel costs are 748.55 to $2736.93 \&$ shown by blue lines.For unit 2 , fuel costs are 946.55 to 1465 for power intervals \& shown by red lines. For unit 3, fuel costs are 745.18 to 1536.83 for power ranges \& shown by green lines.

\section{B. Results obtained for ELD using FL}

Figure 5.2.1 shows fuzzy input membership functions. Membership function referred as "gaussmf" used for three linguistic variables low, medium, high for input variables. Figure shows input variable as high with a range of 0 to 150.

1) Fuzzy input (power) membership function

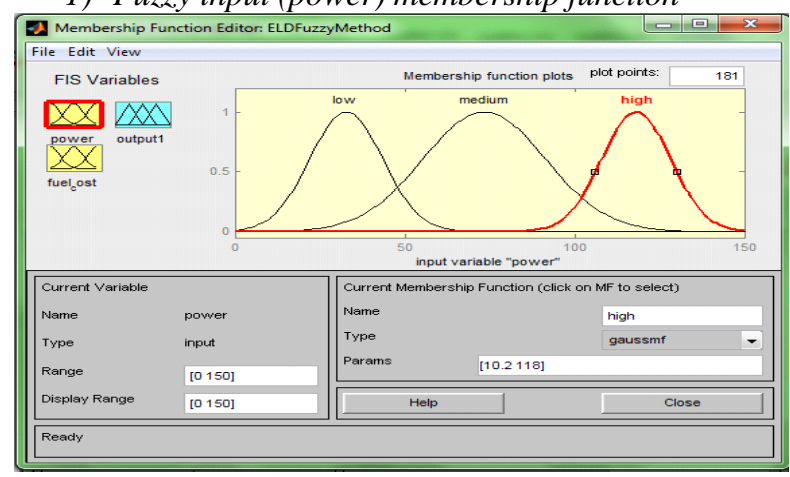

Fig. 3. Fuzzy input (power) membership function

\section{2) Fuzzy output membership function}

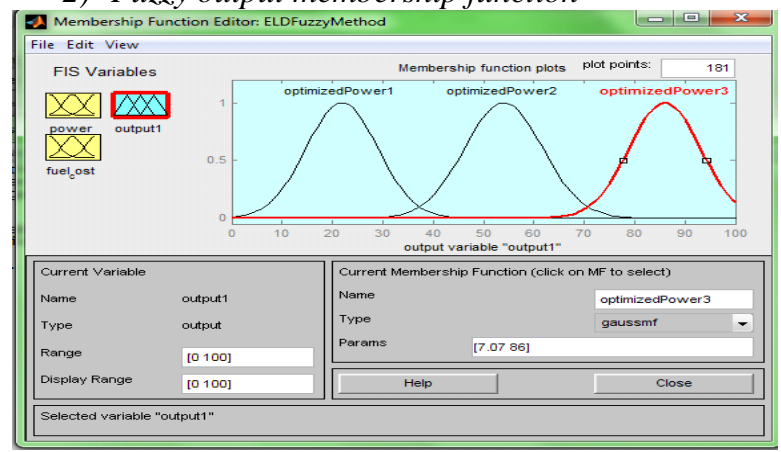

Fig. 4. Fuzzy output membership function

\section{3) Linguistic Variables}

TABLE I. LINGUISTIC LEVELS OF POWER INPUT

\begin{tabular}{|l|l|l|l|}
\hline Input Parameters & Low & Medium & High \\
\hline P(Power) in MW & $0-60$ & $30-130$ & $80-150$ \\
\hline Fuel cost (in Rs) & $0-50$ & $30-70$ & $60-100$ \\
\hline
\end{tabular}

Three membership functions generated for input variable in fuzzy system. The low, medium and high are linguistic levels of power input. For input parameter of power low range is 0 to 60 , medium range is 30 to $130 \&$ high range is 80 to 150 . For fuel cost low range is 0 to 50 , medium range is 30 to $70 \&$ high range is 60 to 100 .

TABLE II. LINGUISTIC LEVELS OF OUTPUT VARIABLES

\begin{tabular}{|l|l|l|l|}
\hline $\begin{array}{l}\text { Output } \\
\text { Parameters }\end{array}$ & $\begin{array}{l}\text { Optimized } \\
\text { Power1 }\end{array}$ & $\begin{array}{l}\text { Optimized } \\
\text { Power2 }\end{array}$ & $\begin{array}{l}\text { Optimized } \\
\text { Power3 }\end{array}$ \\
\hline $\begin{array}{l}\text { Optimized } \\
\text { Power (in MW) }\end{array}$ & $0-42$ & $30-80$ & $65-100$ \\
\hline
\end{tabular}

Table no. 2 shows linguistic levels for output variables. Here, Optimizedpower1, optimizedpower2 and optimizedpower3 are linguistic levels. Optimized power1 computed from 0 to 42 , optimized power 2 computed is 30 to $80 \&$ optimized power 3 computed as 65 to 100 . 
4) Surface Viewer

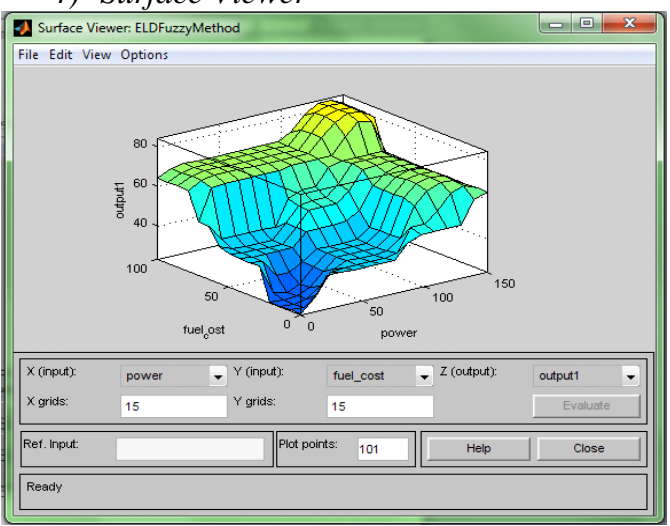

Fig. 5. Surface Viewer

Figure no.5 shows surface viewer is a GUI tool used for examine output surface of a FIS which is stored in a file for any inputs. It selects the two input variables to allot two input axes $\mathrm{Y} \& \mathrm{X}$, this output value allotted to Z-axis. For a smoother plot creation, Plot point field is used to state number points so that membership functions calculated in input/output sort. Here, 101 is a default value which utilized to observe reliance of outputs for any input.Here, input axis shows as power \& fuel cost \& output axis as optimized power.

\section{Results obtained for ELD using Genetic Algorithm} infused with Fuzzy Logic

TABLE III. OPTIMAL Result OBTAINED USING GA

\begin{tabular}{|l|l|l|l|l|}
\hline $\begin{array}{l}\text { Pmin } \\
(\mathrm{PW})\end{array}$ & $\begin{array}{l}\text { Pmax } \\
(\mathrm{MW})\end{array}$ & $\begin{array}{l}\text { Power } \\
\text { generated } \\
(\mathrm{MW})\end{array}$ & $\begin{array}{l}\text { Fuel cost } \\
(\mathrm{Rs})\end{array}$ & Error $(\%)$ \\
\hline 50 & 150 & 50 & 772.5 & 0.1450 \\
75 & 200 & 61.932 & 997.3 & 0.2131 \\
\hline
\end{tabular}

TABLE IV. OPTIMAL RESUlt OBTAINED USING FL

\begin{tabular}{|l|l|l|l|l|}
\hline Pmi (MW) & $\begin{array}{l}\text { Pmax } \\
\text { (MW) }\end{array}$ & $\begin{array}{l}\text { Power } \\
\text { generated } \\
\text { (MW) }\end{array}$ & $\begin{array}{l}\text { Fuel cost } \\
\text { (Rs) }\end{array}$ & Error (\%) \\
\hline 50 & 150 & 69.24 & 779 & 0.105 \\
75 & 200 & 81.321 & 1009 & 0.167 \\
\hline
\end{tabular}

TABLE V. OPtimal Result ObTAINED USING FCGA

\begin{tabular}{|l|l|l|l|l|}
\hline $\begin{array}{l}\text { Pmin( } \\
\text { M) }\end{array}$ & Pmax(MW) & $\begin{array}{l}\text { Power } \\
\text { generated } \\
(\mathrm{MW})\end{array}$ & $\begin{array}{l}\text { Fuel cost } \\
(\mathrm{Rs})\end{array}$ & Error (\%) \\
\hline 50 & 150 & 93.23 & 669.5 & 0.087 \\
75 & 200 & 117.324 & 869.2 & 0.102 \\
\hline
\end{tabular}

To acquire optimal solution for ELD system, comparison of all results done using minimum \& maximum power limits. Here, generated power, cost of fuel \& transmission errors compared to achieve optimal solution. For powers of $50 \mathrm{MW}$ to $150 \mathrm{MW}$, following observations obtained:

1) In GA, generated power is $50 \mathrm{MW}$ for a fuel cost of Rs.772.5 \& an error of 0.14501.In FL method, generated power is $69.24 \mathrm{MW}$ with a fuel cost of Rs.779 \& an error of 0.10501 . In FCGA method, generated power is 93.23 with a fuel cost of $669.5 \&$ an error of 0.00871 . By comparing three parameters (as shown in figures nos.6,7 \& 8) it is found that FCGA method generates more power with less fuel rates $\&$ transmission errors as compared to GA \& FL methods.

2) For minimum power of $75 \mathrm{MW} \&$ maximum power of $200 \mathrm{MW}$, by comparing values in figure nos.6,7 \& 8, it is again found that FCGA methods generates more power with less cost $\&$ transmission errors as compared to GA \& FL methods.

\section{Comparisons of soft computing methods to obtain optimal solution for ELD system}

Comparison of power generation, fuel cost \& errors depicted in figures for three methods.

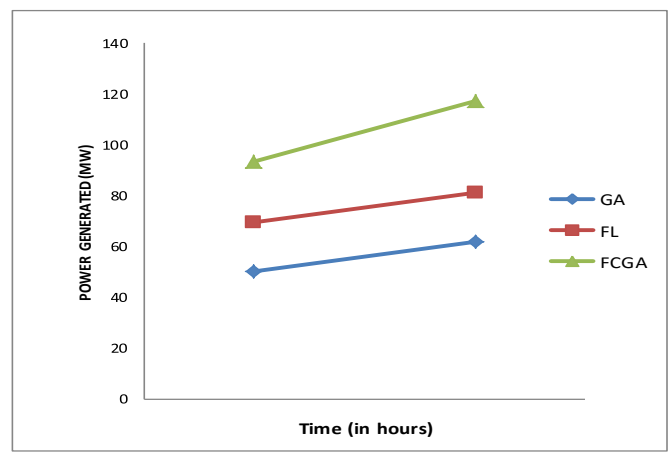

Fig. 6. Comparison of power generation

Figure shows that FCGA produces higher power levels as compared to FL \& GA methods.

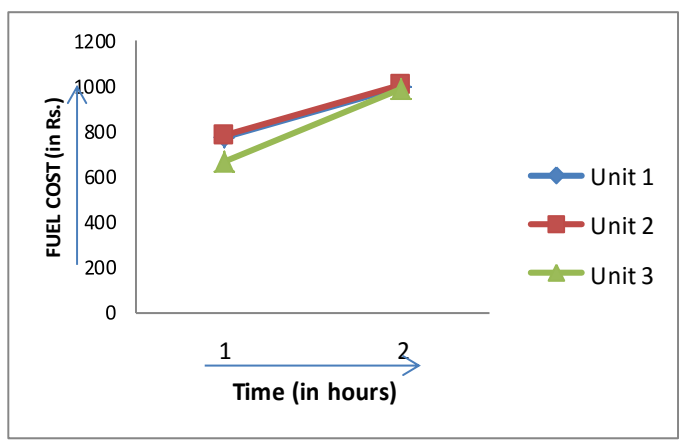

Fig. 7. Comparison of Fuel Costs

Fig.7. shows that FCGA has less fuel cost for power generation as compared to FL \& GA methods. 


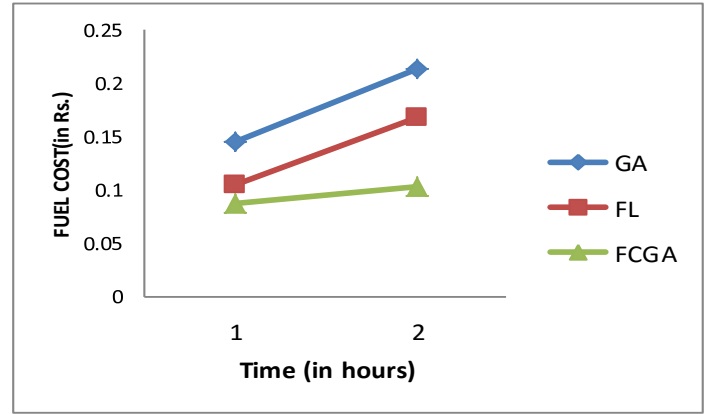

Fig. 8. Comparison of transmission errors

Figure no. 8 shows, FCGA has less transmission errors for power generation as compared to FL \& GA methods.

\section{CONCLUSION}

Simulation results using GA, FL \& GA infused fuzzy shows performance improvement between these algorithms. Results shows that GA infused fuzzy shows has better performance as compared to GA \& FL techniques since FCGA considers average fitness, change in fitness, optimized crossover probability \& optimized mutation probability variables.It observed that performance improvement of ELD increased as required minimum \& maximum power ranges increased, resulting cost of power generation reduces with an increase of power generation using soft computing techniques. Transmission errors reduced to -0.0579 which is $39.93 \%$ less than GA method \& -0.0179 which is $17.04 \%$ less as compared to FL method.So, performance improvement of ELD system is able to increase subsequently by reducing transmission error during generation of power. FCGA proved best for optimal solution of power generation with minimum cost \& transmission errors as compared to GA \& FL methods.

\section{REFERENCES}

[1] Sarat Kumar Mishra, Sudhansu Kumar Mishrab, "A Comparative Study of Solution of Economic Load Dispatch Problem in Power Systems in the Environmental Perspective",International Conference on Intelligent Computing, Communication \& Convergence (ICCC-2014), Procedia Computer Science 48 ( 2015 ) 96 - 100, ELSEVIER

[2] Bin XU, Ping-an ZHONG, Yun-fa ZHAO, Yu-zuo ZHU, Gao-qi ZHANG, "Comparison between dynamic programming and genetic algorithm for hydro unit economic load dispatch", Water Science and Engineering, 2014, 7(4): 420-432 doi:10.3882/j.issn.16742370.2014.04.007

[3] R. Gopalakrishnan and A. Krishnan, "An efficient technique to solve combined economic and emission dispatch problem using modified Ant colony optimization", Sadhana Vol. 38, Part 4, August 2013, pp. 545556, Indian Academy of Sciences

[4] Sunil Kumar Singh, Lobzang Phunchok, Khwairakpam Chaoba singh, Y R Sood, "Genetic Algorithm a Noble Approach For Economic Load Dispatch", International Journal of Engineering Research and Applications (IJERA) ISSN: 2248-9622, National Conference on Emerging Trends in Engineering \& Technology (VNCET-30 Mar'12)

[5] Nagendra Singh and Yogendra Kumar, "Multiobjective Economic Load Dispatch Problem Solved by New PSO", Hindawi Publishing Corporation Advances in Electrical Engineering Volume 2015, Article ID 536040, 6 pages, 10.1155/2015/536040

[6] V.Karthikeyan, S.Senthilkumar \& Vijayalakshmi, "A New Approach to the Solution Of Economic Dispatch Using Particle Swarm Optimization With Simulated Annealing", International Journal on Computational Sciences \& Applications (IJCSA) Vol.3, No.3, June 2013
[7] Ali Hasan Zade, Sayyed Mohammad Ali Mohammadi, Ali Akbar Gharaveisi, "Fuzzy logic controlled differential evolution to solve economic load dispatch problems", Received: 2011/08/07; Accepted: 2011/09/15 Pages: 29-40

[8] Er. Mukesh Garg, Er. Manjeet Singh, Er. Vineet Girdher, "Comparative Study of Economic Load Dispatch (Eld) Using Modified Hopfield Neural Network", Proceedings of 'I-Society 2012' at GKU, Talwandi Sabo Bathinda (Punjab), International Journal of Computing \& Business Research ISSN (Online): 2229-6166,

[9] Paolo Dadone, "Design Optimization of Fuzzy Logic Systems", Dissertation submitted to the Faculty of the Virginia Polytechnic Institute and State University

[10] S. H. Elyas, P. Mandal, A. U. Haque, A. Giani, Tseng Tzu-Liang, "A new hybrid optimization algorithm for solving economic load dispatch problem with valve-point effect", North American Power Symposium (NAPS), pp. 1-6, 2014.

[11] François Chevrie, François Guely, "Fuzzy Logic", Groupe Schneider, "Technical series"

[12] Harpreet Singh, Madan M. Gupta, Thomas Meitzler, Zeng-Guang Hou, Kum Kum Garg, Ashu M. G. Solo, and Lotfi A. Zadeh, "Real-Life Applications of Fuzzy Logic", Advances in Fuzzy Systems, Volume 2013 (2013), Article ID 581879, 3 pages, 10.1155/2013/581879

[13] Bhagyashree Hosamani, C R Sharada Prasad, "Analysis of Economic Load Dispatch Using Fuzzified PSO", IJRET : International Journal of Research in Engineering and Technology eISSN: 2319-1163 | pISSN: 2321-7308

[14] Bishnu Sahu, Soumya Das, T. Manoj Patra, "Economic Load Dispatch in Power System using Genetic Algorithm", International Journal of Computer Applications (0975 - 8887) Volume 67- No.7, April 2013

[15] Gajendra Sahu, Kuldeep Swarnkar, "Economic Load Dispatch by Genetic Algorithm in Power System", International Journal of Science, Engineering and Technology Research (IJSETR), Volume 3, Issue 8, August 2014 ISSN: $2278-7798$

[16] A. Bhattacharya and P. K. Chattopadhyay, "Solving complex economic load dispatch problems using biogeography-based optimization," Expert Systems with Applications, vol. 37, no. 5, pp. 3605-3615, 2010.

[17] X. Xia, A.M. Elaiw, "Optimal dynamic economic dispatch of generation: A review", Electric Power Systems Research, (Elsevier),pp.975-986,2010

[18] S.C. Swain, S. Panda, A.K. Mohanty, C. Ardil,"Application of Computational Intelligence Techniques for Economic Load Dispatch" World Academy of Science, Engineering and Technology pp 63-69, 2010.

[19] F. Farheen, M. A. Ansari, M. Kardam, "Implementation of particle swarm optimization for dynamic economic load dispatch problem", International Conference on Energy Efficient Technologies for Sustainability (ICEETS), pp. 1273-1278, April 2013.

[20] Dubey Manisha, Dubey Aalok ,2010“Genetic Algorithm Based Design of Fuzzy Logic Power System Stabilizers in Multimachine Power System", World Academy of Science, Engineering and Technology vol.66, pp.975-982.

[21] Cho Sung-Bae, 2002"Fusion of neural networks with fuzzy logic and genetic algorithm", Department of Computer Science, Yonsei University, pp.363-372.

[22] Guo-Li Zhang ; Hai-Yan Lu ; Geng-Yin Li ; "Guang-Quan ZhangDynamic economic load dispatch using hybrid genetic algorithm and the method of fuzzy number ranking", Machine Learning and Cybernetics, 2005. Proceedings of 2005 International Conference on (Volume:4 ), 18-21 Aug. 2005, Page(s): 2472 - 2477 Vol. 4, IEEE

[23] T. Yalcinoz, H. Altun, and M. Uzam, "Economic Dispatch Solution Using A Genetic Algorithm Based on Arithmetic Crossover", IEEE

[24] Prof. Vikrant Sharma, Navpreet Singh Tung, Rashmi Rana and Vivek Guleria, "Fuzzy Logic Controller Modelling for Economic Dispatch and Unit Commitment in Electrical Power System", International Journal of Emerging Research in Management \&Technology ISSN: 2278-9359 (Volume-2, Issue-10)

[25] J. Nanda, R. Badri Narayanan, "Application of genetic algorithm to economic load dispatch with Lineflow constraints", Electrical Power and Energy Systems 24 (2002) 723-729 
[26] Bouktir.T, Mahdad.B, and Srairi.K., "Fuzzy Controlled Genetic Algorithm For Envirenmental/Economic Dispatch with Shunt FACTS Devices", IEEE press, pp.201, 2003

[27] Benasla.L, Belmadant.A and Rahli M., "Hooke-Jeeves'Method Applied to a New Economic Dispatch Problem Formulation" journal of information science and engineering 24, 907-917 (2008)

[28] Song Y.H., Wang G.S., Wang P.Y. and Johns A.T., "Environmental economic dispatch using fuzzy logic controlled genetic algorithms," IEE Proc. Generation Transmission Distribution, vol. 144, pp. 377-382, July 1997.

[29] Lily Chopra, Raghuwinder Kaur, "Economic Load Dispatch Using Simple And Refined Genetic Algorithm", International Journal of Advances in Engineering \& Technology, ISSN: 2231-1963, Nov. 2012

[30] Farooqi M.R., Jain P. and Niazi K.R., "Using Hopfield Neural Network for Economic Dispatch of Power Systems", IEEE Power Engineering Conference, 2003. PECon 2003, Proceedings, Bangi, Malaysia, pp. 5-10, 15-16 Dec. 2003.

[31] Park J.H., Kim Y.S., Eom I.K. and Lee K.Y., "Economic Load Dispatch for iecewise Quadratic Cost Function Using Hopfield Neural Network", IEEE Transactions on Power Systems, vol. 8, no. 3, pp. 1030-1038, Aug.1993.

[32] AL-Kandari A.M. and El-Naggar K.M., "A genetic-based algorithm for optimal estimation of input-output curve parameters of thermal power plants," Springer Berlin / Heidelberg Journal of Electrical Engineering, vol. 89, no. 8, pp. 585- 590, Sept., 2007.

[33] Roa C.A-Sepulveda, Herrera M., Pavez-Lazo. B, Knight U.G., Coonick A.H., "Economic Dispatch using fuzzy decision trees", Electric Power Systems Research 66 (2003) 115_/122, Dec 2002

[34] King T.D., El-Hawary M.E. and El-Hawary F., "Optimal environmental dispatching of electric power systems via an improved hopfield neural network model." IEEE Transactions on Power Systems, vol. 10, no. 3, pp.1559-1565, Aug. 1995.

[35] M. Sailaja Kumari1, M.Sydulu, "A Fast Computational Genetic Algorithm for Economic Load Dispatch", International Journal of Recent Trends in Engineering Vol. 1, No. 1, May 2009

[36] Y. Labbi, D. Ben Attous, "A Hybrid GA-PS Method To Solve The Economic Load Dispatch Problem", Journal of Theoretical and Applied Information Technology, 2005 - 2010

[37] R. Ouiddir, M. Rahli and L. Abdelhakem-Koridak, "Economic Dispatch using a Genetic Algorithm: Application to Western Algeria's Electrical Power Network", Journal Of Information Science And Engineering 21, 659-668 (2005)

[38] Leandro dos Santos Coelho, Chu-Sheng Lee, "Solving economic load dispatch problems in power systems using chaotic and Gaussian particle swarm optimization approaches", Electrical Power and Energy Systems 30 (2008) 297-307, ELSEVIER

[39] Hesham K. Alfares and Mohammad Nazeeruddin, "Electric load forecasting: literature survey and classification of methods", International Journal of Systems Science, 2002, volume 33, number 1, pages $23-34$

[40] Altun H. and Yalcinoz T, 2008 "Implementing soft computing techniques to solve economic dispatch problem in power systems," Expert Systems with Applications, vol. 35, pp. 1668-1678
[41] Brar Y.S et.al, 2004 "Interactive fuzzy satisfying multi-objective generation scheduling", Asian Journal of Information Technology, Vol. 3, pp. 973-982.

[42] Casillas Jorge et.al,2007 "Fuzzy-XCS: A Michigan Genetic Fuzzy System", IEEE Transactions on fuzzy systems, vol.15,pp97-107

[43] Chang Y.C et.al,2005 "Optimal chiller loading by genetic algorithm for reducing energy consumption", Energy and Buildings (Elsevier), Vo.37, pp.147-155.

[44] Chawla Gaganpreet et.al,2005 "Artificial Neural Network Applications for Power System Protection", Proceedings of IEEE Canadian Conference on Electrical and Computer Engineering, Saskatoon, Canada, pp. 1954-1957

[45] Mahdad Belkacem et.al,2008“Optimal power Flow of the Algerian Network using Genetic Algorithm/Fuzzy Rules," Power and Energy Society General Meeting - Conversion and Delivery of Electrical Energy in the 21st Century, IEEE, pp. 1-8, 20-24.

[46] Nanda J et.al, 1994 "Economic emission load dispatch with line flow constraints using a classical technique," IET Generation, Transmission and Distribution, vol. 141, no. 1, pp. 1-10

[47] Ozansoy et.al, 2007 "The Real-Time Publisher/Subscriber Communication Model for Distributed Substation Systems", IEEE Transactions on Power Delivery, vol. 22, pp.1411-1423.

[48] Phan Phi Anh and Gale Tim ,2007 "Two-Mode Adaptive Fuzzy Control With Approximation Error Estimator", IEEE Transcations on fuuzy system, vol.15pp. 943-955

[49] Pothiya S et.al ,2008, "Application of multiple tabu search algorithm to solve dynamic Economic Dispatch considering generator constraints", Energy Conservation and Management vol. 49, no. 4, pp. 506-516.

[50] Rabbani M.G et.al ,2004“Application of fuzzy controlled SMES unit in automatic generation control", Department of Electrical and Electronic Engineering Rajshahi University of Engineering \& Technology 3rd International Conference on Electrical \& Computer Engineering ICECE 2004, pp.28-30

[51] Sayah Samir, Zehar Khaled ,2008 "Using Evolutionary Computation to Solve the Economic Load Dispatch Problem, Department of Electrical Engineering, vol. 12, p. 67-78

[52] Coelho L.d.S, Mariani V.C,2009"Chaotic artificial immune approach applied to economic dispatch of electric energy using thermal units,"International Journal of Chaos, Solitons and Fractals( Elsevier), pp 2376-2383.

[53] Alireza Gharegozi and Rozbeh Jahani, "A New Approach for Solving the Unit CommitmentProblem by Cuckoo Search Algorithm", Indian Journal of Science and Technology | Print ISSN: 0974-6846 | Online ISSN: 0974-5645, Vol 6 (9) | September 2013

[54] Bakirtzis. A, Petridis.V, and Kazarlis.S, july 1904 "Genetic Algorithm solution to the Economic Dispatch problem",IEE Proc-Gener, vol.141, No.4

[55] A. G. Bakirtzis, P.N. Biskas, C. E. Zoumas, V. Petridis, "Optimal power flow by enhanced genetic algorithm, IEEE Trans. On Power Systems, Vol. 17, pp. 229-236, May 2002.

[56] Palanichamy C. and Shrikrishna K. (1991), "Simple algorithm for economic power dispatch" electrical power syst. Res, Vol.21 pp 174153. 